\title{
Decision Making in der Medizin
}

Richard O. Binswanger

Dr. med., Mitglied FMH

\author{
Bayes-Theorem, Prävalenz, Prognose und Behandlungsaussichten am Beispiel der \\ Über- und Unterdiagnostik in der Radiologie.
}

Als ich jung Chefarzt wurde, kam zur Freude auch ein Schock. Ich war plötzlich allein verantwortlich. Für jeden einzelnen Befund meiner Klinik und dessen Konsequenzen für den Patienten. Ich war in einem Dilemma: In meiner Assistentenzeit wurde ich zur Überdiagnostik erzogen. Das bedeutete, dass man sehr leicht den Verdacht auf eine schwere Erkrankung, zum Beispiel Krebs, äussern sollte. Das hatte viele unnötige Zusatzuntersuchungen zur Folge. In meiner Oberarztzeit in den Niederlanden lernte ich die genau gegenteilige Haltung kennen. Unterdiagnostik war gefragt. Man durfte nur bei grossem Verdacht eine Diagnose vermuten. Was sollte nun mein eigener Weg werden? Ich fühlte, dass beide Haltungen unvollkommen waren. Es musste einen besseren Mittelweg geben. Also begann ich, mich mit Medical Decision Making zu befassen, und fand für mich die Lösung.

\section{Das Bayes-Theorem}

Thomas Bayes war Geistlicher und Mathematiker. Er lebte von 1702 bis 1761 in England [1].

Sein Theorem berücksichtigt bei Entscheidungen die Prävalenz einer Erkrankung sowie die Sensitivität und Spezifität eines Testverfahrens. Dabei ist neben einer hohen Sensitivität vor allem die hohe Spezifität (Anzahl der falsch positiven Befunde) relevant. Die Tests für das neonatale Screening auf Schilddrüsenunterfunktion haben eine Sensitivität und Spezifität von nahezu hundert Prozent. Deshalb eignen sie sich für die Früherkennung dieser sehr seltenen Erkrankung, obwohl immer noch mehr falsch als richtig positiv sind. Alle Resultate werden deshalb mit einer zweiten Methode überprüft. Radiologische Methoden sind schon dann sehr gut, wenn sie eine Sensitivität und Spezifität von etwa 90 Prozent aufweisen. Auch bei hoher Prävalenz einer Erkrankung liefern sie neben zahlreichen falsch negativen immer noch viel mehr falsch positive als korrekte Befunde, wie das Mammographiescreening zeigt. Radiologische Methoden eignen sich deshalb keinesfalls für das Screening.

\section{Outcomeorientierung}

Das allein ist nicht ausreichend. Es gibt zwei Situationen: Eine schwere Krankheit und eine leichte, und bei beiden muss unterschieden werden nach der Prognose sowie der Gefahr der Abklärung und Behandlung. Zwei Beispiele für die erste Situation: Bei einem fraglichen Befund am Pankreas im Rahmen einer auf eine andere Anomalie ausgeführten Untersuchung darf ich ihn auch als solchen bezeichnen. Die Gründe sind die sehr gefährliche Krankheit und die potentiell schädliche Therapie sowie ausserordentlich schlechte Prognose des Pankreaskarzinoms. Das Risiko, dem Patienten zu schaden, ist viel grösser als die äusserst minimale Chance, ihn zu heilen. Auch können so überflüssige Zusatzuntersuchungen vermieden werden. Dies soll aber im Normalfall dem Patienten und dem Zuweiser mitgeteilt werden, unter Nennung der Begründung. Nebenbefunde, wie zum Beispiel eine Diskusprotrusion bei einer abdominalen Computertomographie, negiere ich überhaupt. Wenn aber auf einem Röntgenbild ein minimaler Hinweis auf eine Osteomalazie erkannt wird, erwähne ich das mit Nachdruck. Denn es ist eine schwere Krankheit, die mit Vitamin D leicht und gefahrlos zu heilen ist und dann eine sehr gute Prognose hat. Ein weiteres Beispiel für die zweite Situation: Bei der Vermutung auf ein Karzinom in einer Mammographie muss Alarm geschlagen werden. Die Primärtherapie ist einfach und nahezu gefahrlos, und je kleiner das Karzinom, desto besser ist die Prognose.

\section{Immer gut gefahren}

Mit der Anwendung des Bayes-Theorems, der Berücksichtigung der Prävalenz, der Prognose und der Gefährlichkeit der Behandlung in der Beurteilung von radiologischen Befunden bin ich ein ganzes Berufsleben lang sehr gut gefahren. Der Entscheid über Über- oder Unterdiagnostik war immer leicht. Mit diesem Vorgehen habe ich Tausende von unnötigen Zusatzuntersuchungen, Unannehmlichkeiten für die Patienten und auch Geld gespart. Und vor allem für die Patienten Nutzen gestiftet.

Literatur

1 https://de.wikipedia.org/wiki/Thomas_Bayes 